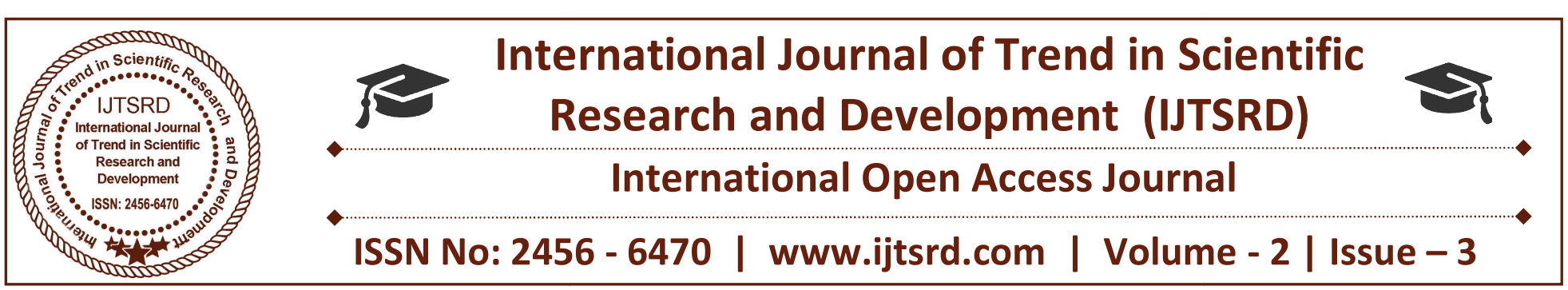

\title{
Location tracking using Google Cloud Messaging on Android
}

\author{
G. Sunderesh Kumar, M. Gokulnath, S. Priya \\ Department of Information Technology, G.K.M. College of Engineering and Technology, \\ Chennai, Tamil Nadu, India
}

\begin{abstract}
Everyone in this world needed to know where is the family member and the most common way is -Make A Call To Each One - Is this a feasible way of doing ? What if someone is busy, or driving a vehicle, or he/she not able to take that call right now, so in that case what will you do drop a message (WhatsAPP) is this what you thinking about then again think about it. You call a person he/she not taking your call then you drop a message to theme again - NO REPLY - but you desperately want to know his/her current location and you again and again call him or message but many times we are in some situation in which we are not able to reply that call/message and we also think that the person should know where am i. Today many people have smartphone running latest android operating system which can be useful to track them and to know their locations. In contrast to traditional solutions, where the calling to the person and asking for their locations is not a feasible and reliable solution. Aim of this application is to track the members by person sitting at home. This paper presents solution to track the locations and communicate with the members using Google MAPs API, GCM (Google Cloud Messaging) and Android Operating System.
\end{abstract}

Keywords: GPS, Point-of-Interest based, FriendFinding, People-Discovery

\section{INTRODUCTION}

The Google maps API are used to show all the devices on that are registered in web application which is running on your home. The smartphone contains android app which sends data at user define period to the web application. This tracking system especially designs for parents to monitor their children's on the move. Consider a situation where a child lost a way to home or he or she meet with an accident in that case child use their tracker app to send alert notification to parents and the web application display it. The web application is design to handle various types of alert that broadcast from android app. We investigate the following fundamental question how fast can we track a family member? And the answer that we got is by calling him but this is not a feasible or reliable solution. Many times it's happen that you're driving and you get the call but you're in condition that you can't answer that call. Now Think about a situation where you found that you lost something or simply you lost your way and then you make a call to someone at home but unfortunately he can't answer your call so what will you do ? Try calling him again and again $80 \%$ of people do this while other try to ask some other person or ask any other in that are

\section{RELATED WORKS}

2005-2006(February):-Google Maps goes live. Just two months later, we add satellite view sand directions to the product. (April):-Google Maps comes to mobile phones in the U.S., offering driving directions and local information to people on the go. Our first Google Maps release in Europe is geared to U.K. users. France, Germany, Italy and Spain follow in 2006.

Today, we offer driving directions in 190+ countries around the world. (June):-Google Mobile Web Search is released, specially formulated for viewing search results on mobile phones. We unveil Google Earth, a 
satellite imagery-based mapping service that lets you take a virtual journey to any location in the world. Google Earth has since been downloaded more than 1 billion times. The Google Maps API is released; developers can embed Google Maps on many kinds of mapping services and sites. Today there are 1 million active websites and apps using the API, reaching 1 billion people every week.

2007:-We add traffic information to Google Maps for $30+$ cities around the U.S. Today, live traffic data is available in $50+$ countries, covering highways, streets and more in $600+$ major cities

\section{PROPOSED SYSTEM}

This paper studies the applicability of PPLP in the setting of mobile apps. We categorize popular location social apps and analyze the trade-offs of privacy and functionality with respect to PPLP enhancements. To investigate the practical performance trade-offs, we present an in-depth case study of an Android application that implements Inner Circle, a state-of-the-art protocol for privacypreserving location proximity. This study indicates that the features of PPLP fits several scenarios of realworld LBS and that the performance of such protocols is, for coarse-grained precision, comparable to real applications

\section{ARCHITECTURE DIAGRAM}
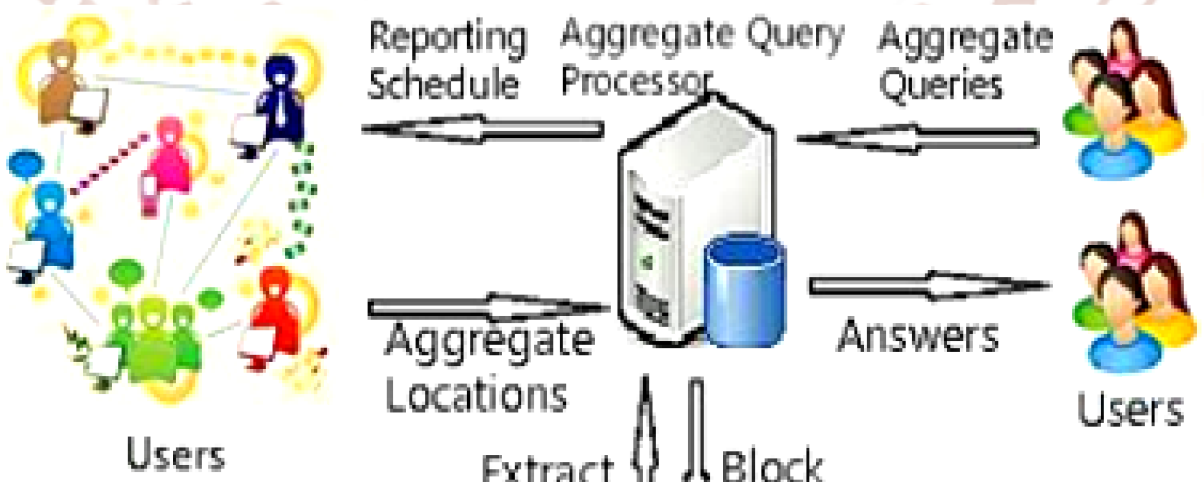

\section{-}

We define three app categories

Point-of-Interest based (PoI), Friend-Finding (FF), and People-Discovery (PD)

PoI apps are common venue-locator applications, e.g. where people wish to meet each other or find a shop of some kind. Friend-Finding apps are for keeping track of the whereabouts of close friends and family. People-Discovery apps are for locating new people to interact with.

\section{ADVANTAGES OF PROPOSED SYSTEM:}

We evaluate to what extent a state-of-the-art protocol can be applied to mobile applications without limiting their functionality

The study uses popular location based social apps from the Google Play Store trade-offs by performance measurements of an implementation of Inner Circle a state-of-the-art privacy-preserving location proximity protocol in an Android application. The study compares the performance of the implementation to real-world applications 
supports with the help of bootstrap frame work. The landing page present with the google map and side menu bar with the following menus. Locate Member To Locate a member on Map Add New Member To register new member Send Message To member To Send push notification on member device app View Notifications To view all messages received from the gtrack app Search A Place To Search a place on map using Google Map Search Box B. Locate Member The locate member is simply shows a popup dialog with all the members name in a drop down. Once we select a member and click on locate button it will move maps to member marker position. C. Add new Member This will shows a popup with the forms to with sets of fields that needed to fields in order to add the member

\section{A. MODULES}

- Firebase Configurations

- Email And Password Authentication

- Location Viewer

- Status Monitoring

\section{B. MODULES DESCRIPTION:}

\section{FIREBASE CONFIGURATIONS:}

There is the firebase configuration we need firebase config file initially the android app package name and version code and authentication with firebase database

\section{EMAIL AND PASSWORD AUTHUNTICATION:}

The next step we authenticate with email and password also insert the user informations into the firebase database

\section{LOCATION VIEWER}

Admin can view the current location of the user by user context while logged on the portal. The context locations of the user in provided by location based service(LBS). this service is accessed by made communication between our mobile phone and cloud database

\section{STATUS MONITORING}

This location is find by the admin from cloud database by using Location based service. Admin can monitor the user location and logged on status.

\section{Conclusions}

This paper determines that applications on an Android device don't need to be running to receive messages. The system will wake up the application via a mechanism called Intent Broadcast when the message arrives, as long as the application is set up with the proper broadcast receiver and permissions. GCM does not provide any built-in user interface or other handling for message data. Instead, it simply passes raw message data received straight to the application, which has full control of how to handle it. For example, the application might post a notification, display a custom user interface, or silently sync data

\section{REFERENCES}

1) G. Dissanayake, S. Sukkarieh, E. Nebot, and H. Durrant-Whyte, "Theaiding of a low-cost strapdown inertial measurement unit using vehiclemodel constraints for land vehicle applications," IEEE Trans. Robot.Autom., vol. 17, no. 5, pp. 731-747, Oct. 2001.

2) H. Xiong and J. Cheng, "Investigation of shortrange high precision $3 \mathrm{D}$ localization via UWB radio," in Proc. IEEE Global Commun. Conf.,Austin, TX, USA, Dec. 2014, pp. 40904095.

3) J. Wang, X. Liang, W. Shi, Q. Niu, T. A. Gulliver, and X. Liu, "Fingerprinting localization based on $60 \mathrm{GHz}$ impulse radio," in Proc.IEEE Pacific Rim Conf. Commun. Comput. Signal Process., Aug. 2015,pp. 491-495.

4) Y. Shen and M. Z. Win, "Fundamental limits of wideband localization-Part I: A general framework," IEEE Trans. Inf. Theory, vol.56,no. 10,pp. 4956-4980, Oct. 2010.

5) K. D. Rao, M. N. S. Swamy, and E. I. Plotkin, "GPS navigation withincreased immunity to modeling errors," IEEE Trans. Aerosp. Electron.Syst., vol. 40, no. 1, pp. 2-11, Jan. 2004.

6) H. Xiong, H. Song, Z. Lai, J. Zhang, and K. Yi, "A novel indoor localization scheme," in Proc. IEEE Int. Conf. Commun. Technol.,Nanjing, China, Nov. 2010, pp. 338-341.

7) S. Wang, Z. Deng, and G. Yin, "An accurate GPSIMU/DR data fusion method for driverless car based on a set of predictive models and grid constraints," Sensors, vol. 16, no. 3, p. 280, Mar. 2016.

8) H. Xiong, W. Zhang, Z. Du, B. He, and D. Yuan, "Front-end narrowband interference mitigation for 
DS-UWB receiver," IEEE Trans. Wireless Commun., vol. 12, no. 9, pp. 4328-4337, Sep. 2013.

9) H. Xiong, "An efficient narrowband interference suppression approach in ultra-wideband receiver," IEEE Sensors J., vol. 17, no. 9, pp. 2741-2748, May 2017.
10) H. Xiong, W. Zhang, H. Xu, Z. Du, H. Tang, and J. Li, "A novel complex-coefficient in-band interference suppression algorithm for cognitive ultra-wide band wireless sensors networks," Sensors, vol. 17, no. 6,pp. 1206-1226, May 2017.298 IEEE SENSORS JOURNAL,VOL. 18, NO. 1, JANUARY 1, 2018

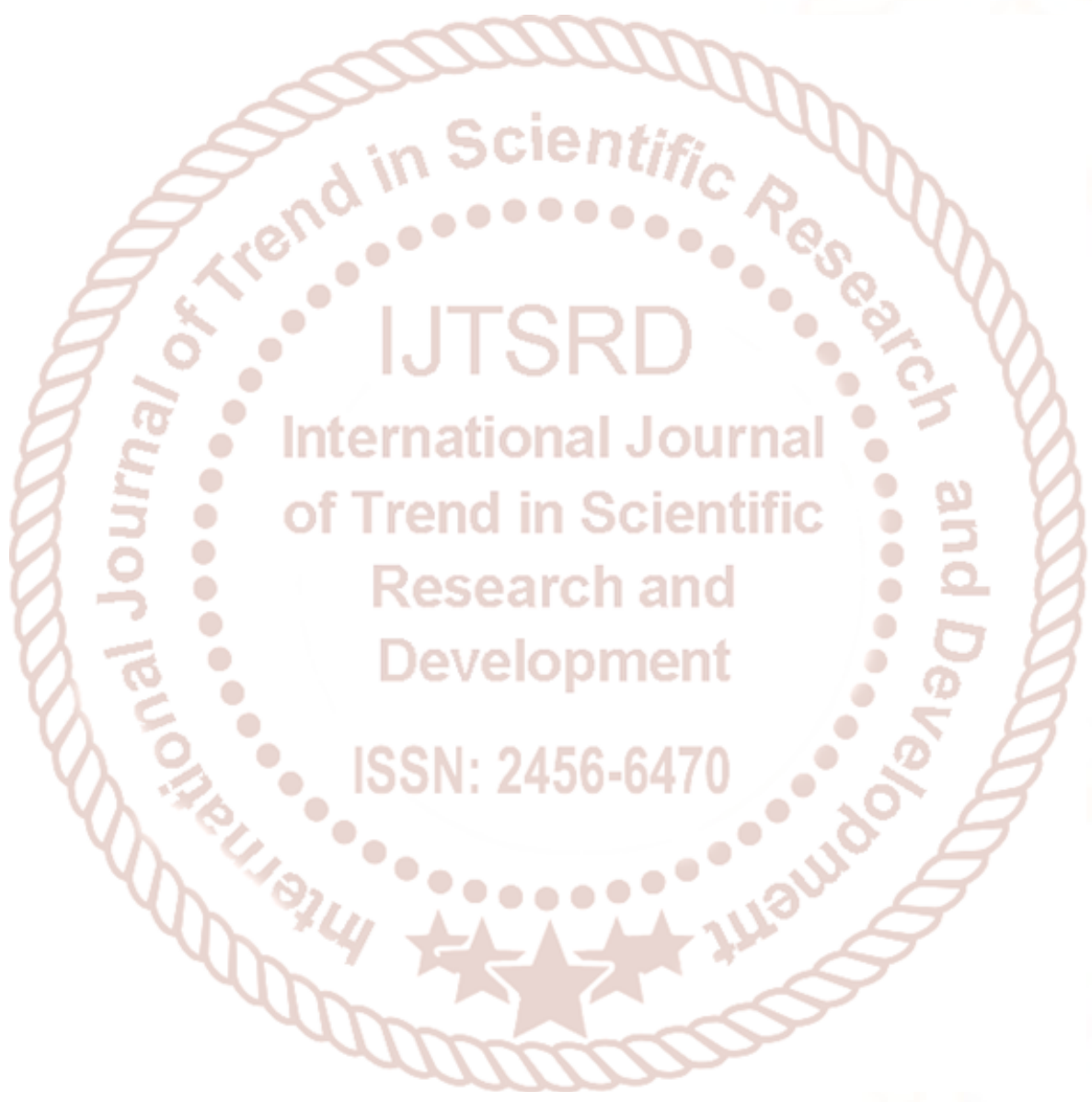

